II. SHIGA AND II. TANIGAWA

KODAI MATH. J.

16 (1993), 361-378

\title{
GRUNSKY'S INEQUALITY AND ITS APPLICATIONS TO TEICHMÜLLER SPACES
}

\author{
Dedicated to Professor Nobuyuki Suita on his sixtieth birthday
}

\author{
By Hiroshige Shiga and Harumi Tanigawa
}

\begin{abstract}
In this paper, we discuss the equality between invariant quantities, such as the Teichmüller distance and the Carathéodory distance, in Teichmüller spaces. We shall give some conditions under which these quantities are the same at some points. Examples of Fuchsian groups will be constructed in relation to the conditions above. In particular, we shall construct a Teichmüller disk in $T(1)$ determined by a quadratic differential with a simple zero where the Teichmüller distance and the Carathéodory distance are the same. We also give an estımate of the variation of critical exponent quasi-Fuchsian groups in terms of Grunsky coefficients.
\end{abstract}

\section{Introduction}

Let $S$ be a hyperbolic Riemann surface. Then there exists a Fuchsian group $\Gamma$ acting on the unit disk presenting $S$. From an identification called the Bers embedding, the Teichmüller space $T(S)$ of $S$ is regarded as a bounded domain $T(\Gamma)$ of the Banach space of bounded holomorphic quadratic differentials for $\Gamma$. Therefore, a natural complex structure of the Teichmüller space is induced via the embedding. Under this complex structure, various analytic properties of Teichmüller spaces are obtained (cf. [1], [3], [5] etc.). On the other hand, from the construction of the Bers embedding each point of the Teichmüller space is the Schwarzian derivative of a univalent function outside the unit disk. Thus, the method of univalent function theory is useful to study Teichmüller spaces. In fact, Grunsky's inequality for univalent functions yields a lot of properties of Teichmüller spaces (cf. [14], [15], [18]). The main purpose of this paper is to investigate invariant metrics of 'Teichmüller spaces from the view point of the univalent function theory.

First, we shall discuss two invariant distances, the Teichmüller-Kobayashi distance and the Carathéodory distance in relation to Grunsky's inequality. As for these distances, when $S$ is a (hyperbolic) Riemann surface of finite type,

Received August 20, 1992 , revised March 19, 1993. 
namely, $\Gamma$ is a finitely generated Fuchsian group of the first kind, Kra [6] shows that the two distances are the same on Teichmüller disks defined by bounded holomorphic quadratic differentials with zeros of even order only. Recently, Krushkal' [8], using a family of holomorphic functions on $T(1)$ defined by Grunsky's coefficients, has shown that they are also the same on similar Teichmüller disks as above when $\Gamma$ is the trivial group, namely, in the case of the universal Teichmüller space. In this paper we shall give a condition on $\Gamma$ under which such a phenomena occurs, in terms of Poincaré series (Theorem 2.1). (Note that even to elementary groups, Krushkal's result can not be extended immediately, since, in general, Poincaré theta operator gives no information about the orders of zeros of quadratic differentials.) The main tool is the recent development for Poincaré series by C. McMullen [10], [11]. The comparison of the Caratnéodory distance on Teichmüller spaces of elementary groups to that of the universal Teichmüller space will be discussed.

Next, we shall estimate the variation of the critical exponent of Fuchsian groups in the Bers embeddings in terms of the norm of Grunsky inequalities (Theorem 2.2). Our estimate implies the estimate of the Hausdorff dimension of limit sets for finitely generated Fuchsian groups (Corollary 2.2).

In the last section, we shall give examples of holomorphic disks in Teichmüller spaces where the Teichmüller-Kobayashi distance and the Carathéodory distance are the same while the Carathéodory distance is given without using Grunsky's coefficients.

The authors are grateful to the referee for reading carefully the first draft of this paper and pointing out some erroneous arguments.

\section{Preliminaries and main results}

In this paper, we denote by $\Delta$ the unit disk and by $\Sigma$ its exterior $\bar{C}-\bar{J}$ where $\overline{\boldsymbol{C}}=\boldsymbol{C} \cup\{\infty\}$ is the Riemann sphere. For a Fuchsian group $\Gamma$ acting on $\Sigma$ (and $\Delta$ ), we consider the Banach space $B_{2}(\Sigma, \Gamma)$ of bounded holomorphic quadratic differentials for $\Gamma$. Namely, the space $B_{2}(\Sigma, \Gamma)$ is the set of holomorphic functions $\varphi$ on $\Sigma$ satisfying

$$
\varphi(\gamma(z)) \gamma^{\prime}(z)^{2}=\varphi(z) \quad \text { for all } z \in \Sigma \text { and for all } \gamma \in \Gamma
$$

and

with norm

$$
\varphi(z)=o\left(|z|^{-3}\right) \quad \text { near } z=\infty
$$

$$
\|\varphi\|_{\Sigma, \infty}=\sup _{z \in \Sigma} \lambda_{\Sigma}(z)^{-2}|\varphi(z)|<\infty,
$$

where $\lambda_{\Sigma}(z)|d z|$ is the Poincare metric on $\Sigma$.

For each $\varphi \in B_{2}(\Sigma, \Gamma)$ there exists a locally univalent holomorphic function $W_{\varphi}$ on $\Sigma$ such that $W_{\varphi}(z)=z+\sum_{n=1}^{\infty} a_{n} z^{-n}$ near $z=\infty$ and the Schwarzian derivative $\left\{W_{\varphi}, z\right\}=\left(W_{\varphi}^{\prime \prime} / W_{\varphi}^{\prime}\right)^{\prime}(z)-(1 / 2)\left(W_{\varphi}^{\prime \prime} / W_{\varphi}^{\prime}(z)\right)^{2}$ is equal to $\varphi(z)(z \in \Sigma)$. The Teich- 
müller space $T(\Gamma)$ of $\Gamma$ is the set of $\varphi \in B_{2}(\Sigma, \Gamma)$ such that $W_{\varphi}$ admits a $\Gamma$ compatible quasiconformal extension to $\overline{\boldsymbol{C}}$. Since $W_{\varphi} \gamma W_{\varphi}^{-1}$ is still a Möbius transformation for $\gamma \in \Gamma, \Gamma^{\varphi}=W_{\varphi} \Gamma W_{\varphi}^{-1}$ is a Kleinian group. Hence, $T(\Gamma)$ is regarded as the set of such Kleinian groups that are quasiconformal deformations of $\Gamma$. When $\Gamma$ is the trivial group, we call $T(\{i d\}$.$) the universal Teich-$ müller space and denote it by $T(1)$. For any Fuchsian group $\Gamma$ the Teichmüller space $T(\Gamma)$ is a subset of $T(1)$, since $B_{2}(\Delta, 1) \supset B_{2}(\Delta, \Gamma)$.

Take the Grunsky coefficients $b_{m n}(\varphi)$ of $W_{\varphi}$, which are determined by the expansion :

$$
\log \frac{W_{\varphi}(z)-W_{\varphi}(\zeta)}{z-\zeta}=-\sum_{m, n=1}^{\infty} b_{m n}(\varphi) z^{-m} \zeta^{-n}
$$

Then it is known (cf. [4]) that $W_{\varphi}$ is univalent in $\Sigma$ if and only if the inequality, called the Grunsky inequality,

$$
\left|\sum_{m, n=1}^{\infty} \sqrt{\overline{m n}} b_{m n}(\varphi) x_{m} x_{n}\right| \leqq\|x\|^{2}
$$

holds for all $x=\left(x_{1}, x_{2}, \cdots\right) \subseteq l^{2}$, where $\|x\|^{2}=\sum_{n=1}^{\infty}\left|x_{n}\right|^{2}$. It is easy to see that Grunsky coefficients $b_{m n}$ are holomorphic functions on $T(\Gamma)$.

When $W_{\varphi}$ has a quasiconformal extension with the Beltrami differential $\mu$, we denote the quasiconformal mapping of $\overline{\boldsymbol{C}}$ by $w^{\mu}$. Using the variational method of quasiconformal mappings, we obtain the following variational formula of Grunsky coefficients ([8]). Then

Proposition 2.1. Let $\mu$ be a Beltrami coefficient on $\overline{\boldsymbol{C}}$ which vanishes in $\Sigma$.

$$
b_{m n}[t \mu]=-\frac{t}{\pi} \iint_{\Delta} \mu(z) z^{m+n-2} d x d y+o\left(\left|t^{1}\right|\right) \quad(t \rightarrow 0),
$$

where $\left\{b_{m n}[t \mu]\right\}_{m, n=1}^{\infty}$ are Grunsky coefficients of $w^{t \mu}$.

Let $\varphi \in T(\Gamma)$ such that $W_{\varphi}$ has a $K$-quasiconformal extension to $\overline{\boldsymbol{C}}$. Then, it is known that

$$
\left|\sum_{m, n=1}^{\infty} \sqrt{m n} b_{m n}(\varphi) x_{m} x_{n}\right| \leqq \frac{K-1}{K+1}\|x\|^{2}
$$

We set

$$
\kappa(\varphi)=\sup _{\|x\|=1}\left|\sum_{m, n=1}^{\infty} \sqrt{m n} b_{m n}(\varphi) x_{m} x_{n}\right| .
$$

From the above inequality, we see that

$$
\rho(0, \kappa(\varphi)) \leqq \tau_{T}(0, \varphi) \leqq \tau_{T(\Gamma)}(0, \varphi),
$$

where $\rho(\cdot, \cdot)$ is the Poincaré distance in $\Delta, \tau_{T}(\cdot, \cdot)$ and $\tau_{T(\Gamma)}(\cdot, \cdot)$ are the 
Teichmüller distances in $T=T(1)$ and in $T(\Gamma)$, respectively.

We define another Banach space $A_{1}(\Delta, \Gamma)$ of all integrable holomorphic quadratic differentials for $\Gamma$ in $\Delta$. The space $A_{1}(\Delta, \Gamma)$ consists of holomorphic functions $\phi$ in $\Delta$ such that

$$
\phi(\gamma(z)) \gamma^{\prime}(z)^{2}=\phi(z) \quad \text { for all } z \Subset \Delta \text { and for all } \gamma \Xi \Gamma
$$

and

$$
\|\phi\|_{\Delta / \Gamma, 1}=\iint_{\Delta / \Gamma}|\phi(z)| d x d y<+\infty .
$$

The Poincaré theta operator $\Theta_{\Gamma}$, defined by

$$
\Theta_{\Gamma}(\phi)(z)=\sum_{\gamma \in \Gamma} \phi(\gamma(z)) \gamma^{\prime}(z)^{2},
$$

is a bounded operator of $A_{1}(\Delta, 1)$ onto $A_{1}(\Delta, \Gamma)$. The norm $\left\|\Theta_{\Gamma}\right\|$ of $\Theta$ is less than or equal to one. C. McMullen ([10], [11]) shows that for most of Fuchsian groups, $\left\|\Theta_{\Gamma}\right\|<1$. Ohtake [13] characterizes Fuchsian groups $\Gamma$ with $\left\|\Theta_{\Gamma}\right\|=1$ in terms of geometric conditions for $\Delta / \Gamma$.

Here we recall extremal Beltrami coefficients. Let $M(\Delta, \Gamma)$ be the space of all Beltrami coefficients on $\Delta$ for $\Gamma$, namely, Beltrami coefficients such that $\mu \circ \gamma \cdot \bar{\gamma}^{\prime} / \gamma^{\prime}=\mu$ for all $\gamma: \equiv \Gamma$. For each Beltrami coefficient $\mu$ in $\Delta$, we extend $\mu$ to the whole plane by setting 0 outside $\Delta$. Let $w^{\mu}$ be the quasiconformal automorphism of the Riemann sphere with Beltrami coefficient $\mu$ which is normalized at $\infty$ so that

$$
w^{\mu}(\zeta)=\zeta+O\left(|\zeta|^{-1}\right)
$$

near $\zeta=\infty$. The assignment $\mu \rightarrow\left\{w^{\mu}, \cdot\right\}$ induces a holomorphic mapping of $M(\Delta, \Gamma)$ onto $T(\Gamma)$. For each $\mu \in M(\Delta, \Gamma)$, the point in $T(\Gamma)$ determined by $\mu$ is denoted by $[\mu]$.

'Two Beltrami coefficients $\mu, \nu \cong M(\Delta, \Gamma)$ are said to be equivalent (with respect to the equivalence relation for $T(\Gamma)$ ) if they determines the same point in $T(\Gamma)$. A Beltrami coefficient $\mu$ is said to be extremal if its supremum norm $\|\mu\|_{\infty}$ is not greater than that of any other elements in its equivalence class. A Beltrami coefficient $\mu \in M(\Delta, \Gamma)$ is extremal if and only if there exists a sequence $\left\{\phi_{n}\right\}_{n=1}^{\infty} \subset A_{1}(\Delta, \Gamma)$ with $\left\|\phi_{n}\right\|_{\Delta / \Gamma, 1} \leqq 1$ such that $\lim _{n \rightarrow \infty}\left|\iint_{\Delta, \Gamma} \mu \phi_{n}\right|=\|\mu\|_{\infty}$. Such a sequence $\left\{\phi_{n}\right\}_{n=1}^{\infty}$ is called a Hamilton sequence for $\mu$. (See, for example, Lehto [9].) In particular, a Beltrami coefficient of the form $t \bar{\phi} /|\phi|$ with $t \Subset \Delta$ and $\phi \in A_{1}(\Delta, \Gamma)$ is extremal. Such a Beltrami coefficient is called a Teichmüller differential.

For an extremal Beltrami differential $\mu \in M(\Delta, \Gamma)$, we define a holomorphic mapping of $\Delta$ into $T(\Gamma)$ by

$$
F_{\mu}(t)=\left[t \mu /\|\mu\|_{\infty}\right]
$$


for $t \subseteq \Delta$. Then for each $t \Subset \Delta$,

$$
\rho(0, t)=\tau_{T(\Gamma)}\left(0, F_{\mu}(t)\right) .
$$

We shall denote by $\boldsymbol{D}(\mu)$ the image of $\Delta$ by $F_{\mu}$ and call a geodesic disk determined by the extremal Beltrami coefficient $\mu \boxminus M(\Delta, \Gamma)$. For a Teichmüller differential $t \bar{\phi} /|\phi|$, we shall abbreviate $\boldsymbol{D}(t \bar{\phi} /|\phi|)$ by $\boldsymbol{D}(\phi)$ and call it a Teichmüller disk.

By utilizing Grunsky's coefficients, Krushkal' [8] has shown the following;

Proposition 2.2. Let $\mu \boxminus M(\Delta, 1)$ be an extermal Beltrami coefficient. Assume that there exists a Hamilton sequence for $\mu$ consisting of quadratic differentials in $A_{1}(\Delta, 1)$ of even order only. Then the Carathéodory distance is equal to the Teichmüller-Kobayashi distance on $\boldsymbol{D}(\mu)$. In fact,

for all $\varphi \in \boldsymbol{D}(\mu)$.

$$
\rho(0, \kappa(\varphi))=c_{T(1)}(0, \varphi)=\tau_{T(1)}(0, \varphi)
$$

We extend the above result as follows.

THEOREM 2.1. (a) Let $\Gamma$ be an elementary Fuchsian group acting on $\Delta$. Let $\mu \in M(\Delta, \Gamma)$ be an extremal Beltrami coefficient. Assume that there exists a Hamilton sequence for $\mu$ consisting of quadratic differentials in $A_{1}(\Delta, \Gamma)$ with zeros of even order only. Then the Caratheodory distance is equal to the Teichmüller-Kobayashi distance on $\boldsymbol{D}(\mu)$

(b) Let $\Gamma$ be a torsion free Fuchsian group acting on the unit disk $\Delta$. Then there exists $\varphi \in T(\Gamma)$ such that

$$
\rho(0, \kappa(\varphi))=\tau_{T(1)}(0, \varphi)=\tau_{T(\Gamma)}(0, \varphi)
$$

if and only if $\left\|\Theta_{\Gamma}\right\|=1$.

Let $c_{T(\Gamma)}(\cdot, \cdot)$ be the Carathéodory distance on $T(\Gamma)$. Then, by the distance decreasing property,

and

$$
\begin{aligned}
& c_{T(\Gamma)}(p, q) \geqq c_{T(1)}(p, q), \\
& \tau_{T(\Gamma)}(p, q) \geqq \tau_{T(1)}(p, q)
\end{aligned}
$$

$$
\tau_{T(\Gamma)}(p, q) \geqq c_{T(\Gamma)}(p, q),
$$

for $p, q \in T(\Gamma)$. It is known that if $\Gamma$ is an elementary Fuchsian group, then $\tau_{T(\Gamma)}(p, q)=\tau_{T(1)}(p, q)$ for any $p, q \subseteq T(\Gamma)$. We show a similar result for the Carathéodory distance on geodesic disks $\boldsymbol{D}(\mu)$.

COROllary 2.1. Let $\Gamma$ and $\mu$ be as in Theorem 2.1 (a). Then, $c_{T(\Gamma)}=\tau_{T(\Gamma)}$ $=c_{T(1)}=\tau_{T(1)}$ on $\boldsymbol{D}(\mu)$. Furthermore, 
for all $\varphi \in \boldsymbol{D}(\mu)$.

$$
\rho(0, \kappa(\varphi))=c_{T(\Gamma)}(0, \varphi)=\tau_{T(\Gamma)}(0, \varphi)
$$

Next, we shall state our results about critical exponents of an arbitrary Fuchsian group $\Gamma$ acting on $\Delta$.

DEFINITION 2.1. Let $G$ be a Kleinian group. We suppose that $\infty$ is an ordinary point of $G$ and not an elliptic fixed point of $G$. Then the critical exponent $\delta(G)$ of $G$ is defined by

$$
\begin{aligned}
\delta(G) & =\sup \left\{\mu: \sum_{\boldsymbol{g} \in G}|c|^{-2 \mu}=+\infty, g(z)=(a z+b) /(c z+d)(a d-b c=1)\right\} \\
& =\inf \left\{\mu: \sum_{g \in G}|c|^{-2 \mu}<+\infty, g(z)=(a z+b) /(c z+d)(a d-b c=1)\right\} .
\end{aligned}
$$

We estimate $\delta\left(\Gamma^{\varphi}\right)$ in terms of $\kappa(\varphi)$ and $\delta(\Gamma)$.

THEOREM 2.2. Let $\Gamma$ be an arbitrary Fuchsian group acting on $\Delta$. Suppose that $\infty$ is not an elliptic fixed point of $\Gamma$. Then for $\varphi=T(\Gamma)$,

$$
\frac{\delta(\Gamma)}{1+\kappa(\varphi)} \leqq \delta\left(\Gamma^{\varphi}\right) \leqq \frac{\delta(\Gamma)}{1-\kappa(\varphi)}
$$

Sullivan [16] shows that for a geometrically finite Kleinian group the critical exponent is equal to the Hausdorff dimension of the limit set. Thus, we have

COROLlARY 2.2. Let $\Gamma$ and $\varphi$ be as the same ones as above. Furthermore, we assume that $\Gamma$ is finitely generated. Then

$$
\frac{H(\Gamma)}{1+\kappa(\varphi)} \leqq H\left(\Gamma^{\varphi}\right) \leqq \frac{H(\Gamma)}{1-\kappa(\varphi)},
$$

where $H(G)$ is the Hausdorff dimension of the limit set of $G$.

Remark. Put $K(\varphi)=e^{d}$ for $d=\tau_{T(1)}(0, \varphi)$. Since $W_{\varphi}$ is a $K(\varphi)$-quasiconformal mapping in the plane, it is $K(\varphi)^{-1}$ Hölder continuous. Therefore, it is easy to see that

$$
K(\varphi)^{-1} H(\Gamma) \leqq H\left(\Gamma^{\varphi}\right) \leqq K(\varphi) H(\Gamma) .
$$

We know that $K(\varphi) \geqq(1+\kappa(\varphi) / 1-\kappa(\varphi))$ because $\tau_{T(1)}(0, \varphi) \geqq \rho(0, \kappa(\varphi))$. Thus, our estimate is an improvement of the above one.

\section{Proofs of Theorem 2.1 and Corollary 2.1}

Proof of (a). For the sake of simplicity, we shall give a proof in the case of a Teichmüller disk $\boldsymbol{D}(\phi)$ defined by a quadratic differential $\phi \in A_{1}(\Delta, \Gamma)$ with zeros of even order only. The general cases can be proved in the same way. 
If $\Gamma=\langle\gamma\rangle$ is a finite group, then it is an Abelian group and obviously $A_{1}(\Delta, \Gamma) \subset A_{1}(\Delta, 1)$. Thus for $\phi E A_{1}(\Delta, \Gamma)-\{0\}$ with zeros of even order only, the equality $c_{T(1)}=\tau_{T(1)}$ holds on $\boldsymbol{D}(\phi)$ from Proposition 2.2. From the distance decreasing property, we have $c_{T(1)} \leqq c_{T(\Gamma)}$. On the other hand, since $\Gamma$ is an Abelian group, $\tau_{T(1)}=\tau_{T(\Gamma)}$ (cf. [12] Theorem 1). Thus we have $\tau_{T(\Gamma)} \leqq c_{T(\Gamma)}$ and the desired equality. It means also the proof of Corollary 2.1.

We shall show the theorem for elementary groups of infinite order.

LEMMA 3.1. Let $\mu$ be an extremal Beltrami differential for $\varphi \Subset T(\Gamma)$, that is, $\mu$ is a measurable function in $\Delta$ with

$$
\tau_{T(\Gamma)}(0, \varphi)=\log \frac{1+\|\mu\|_{\infty}}{1-\|\mu\|_{\infty}}
$$

and

$$
\mu\left(\gamma(z) \overline{\gamma^{\prime}(z)} \gamma^{\prime}(z)^{-1}=\mu(z),\right.
$$

for all $\gamma \leqq \Gamma$ and for almost all $z \leqq \Delta$. Then, the followings are equivalent:

1. $\rho(0, \kappa(\varphi))=\tau_{T(\Gamma)}(0, \varphi)$.

2. $\sup \left|\iint_{\Delta} \mu \phi\right|=\sup \left|\iint_{\Delta_{I} \Gamma} \mu \Theta_{\Gamma}(\phi)\right|=\|\mu\|_{\infty}$, where the supremum is taken over all $\phi$ which are squares of holomorphic functions on $\Delta$ with $\|\phi\|_{\Delta}=1$.

Proof of Lemma 3.1. This is an immediate consequence of Krushkal [7] which shows that $\kappa(\varphi)=\sup \left|\iint_{\Delta} \mu \phi\right|=\sup \left|\iint_{\Delta_{I} \Gamma} \mu \Theta_{\Gamma}(\phi)\right|$ where the supremum is taken over all functions $\phi$ as above.

Although the similar argument in Krushkal' [7] yields the proof of the following lemma, we give a sketch of the proof because we use the argument and notations later.

LEMMA 3.2. Let $\Gamma$ be a Fuchsian group acting on $\Delta$. Suppose that $\phi \cong$ $A_{1}(\Delta, \Gamma)$ admits a sequence $\left\{\psi_{n}\right\}_{n=1}^{\infty} \subset A_{1}(\Delta, 1)$ such that each $\psi_{n}$ has zeros of even order only, $\Theta_{\Gamma}\left(\phi_{n}\right)=\phi(n=1,2, \cdots)$ and $\left\|\phi_{n}\right\|_{\Delta, 1} \rightarrow\|\phi\|_{\Delta / \Gamma, 1}$ as $n \rightarrow \infty$. Then the statements of Theorem 2.1 (a) and Corollary 2.1. hold on $\boldsymbol{D}(\phi)$.

Proof of Lemma 3.2. From the Schwarz lemma and usual normal family argument of bounded holomorphic functions, for the proof of Theorem 2.1, it is sufficient to show that there is a sequence $\left\{f_{n}\right\}_{n=1}^{\infty}$ of holomorphic functions $f_{n}$ of $T(\Gamma)$ to $\left\{z \equiv C:|z|<r_{n}\right\} \quad(n=1,2, \cdots)$ such that $\lim _{n \rightarrow \infty} r_{n}=1$ and

$$
\lim _{n \rightarrow \infty}\left(f_{n} \circ F_{\phi}^{\prime}\right)^{\prime}(0)=1 \text {, }
$$

where $F_{\phi}(t)=[t \bar{\varphi} /|\varphi|]$.

We may assume that $\|\phi\|_{\Delta / \Gamma_{, 1}=1}$. Since $\left\{\psi_{n}\right\}_{n=1}^{\infty} \subset A_{1}(\Delta, 1)$ be a sequence for 
$\phi$ as above, we have

$$
\lim _{n \rightarrow \infty}\left\|\phi_{n}\right\|_{\Delta, 1}=\|\phi\|_{\Delta / \Gamma, 1}=1 .
$$

Since $\phi_{n} \in A_{1}(\Delta, 1)$ have zeros of even order only, by the argument in Krushkal' [8], we have

$$
\phi_{n}(z)=\sigma_{n}(z)^{2}=\frac{1}{\pi} \sum_{k, l=1}^{\infty} \sqrt{k l} x_{k}^{(n)} x_{l}^{(n)} z^{k+l-2} d z^{2}, \quad z=\Delta
$$

where $\sigma_{n}=1 / \sqrt{\pi} \sum_{k=1}^{\infty} \sqrt{k} x_{k}^{(n)} z^{k-1} d z$ are Abelian differentials for $\Gamma$ in $\Delta$ and $x^{(n)}=\left(x_{1}^{(n)}, x_{2}^{(n)}, \cdots\right) \subseteq l^{2}$. Note that $\left\|x^{(n)}\right\|=\left\|\phi_{n}\right\|_{\Lambda, 1}$.

We define a holomorphic function $f_{n, N}$ of $T(\Gamma)$ by

$$
f_{n, N}(\varphi)=\sum_{k, l=1}^{N} \sqrt{k l} x_{k}^{(n)} x_{l}^{(n)} b_{k l}(\varphi),
$$

where $b_{k l}(\varphi)(\varphi \in T(\Gamma))$ are Grunsky coefficients of $W_{\varphi}$ defined in Sec. 2. The Grunsky inequality implies that

$$
\left|f_{n, N}(\varphi)\right| \leqq \kappa(\varphi)\left\|x^{(n)}\right\|=\kappa(\varphi)\left\|\phi_{n}\right\|_{\Delta, 1}
$$

for every $\varphi \in T(\Gamma)$.

From Proposition 2.1, we have

$$
\left(f_{n, N} \circ F_{\phi}\right)^{\prime}(0)=-\sum_{k, l=1}^{N} \frac{1}{\pi} \iint_{\Delta} \frac{\bar{\phi}}{|\phi|} \sqrt{k l} x_{k}^{(n)} x_{l}^{(n)} z^{k+l-2} d x d y .
$$

Since $\bar{\phi} /|\phi|$ is a Beltrami coefficient for $\Gamma$,

$$
\begin{aligned}
\left(f_{n, N} \circ F_{\phi}\right)^{\prime}(0) & =-\frac{1}{\pi} \sum_{k, l=1}^{N} \iint_{\Delta_{l} \Gamma|\phi|} \frac{\bar{\phi}}{|\phi|} \sqrt{k l} x_{k}^{(n)} x_{l}^{(n)} \Theta_{\Gamma}\left(z^{k+l-2}\right) \\
& =-\iint_{\Delta_{l} \Gamma|\phi|} \frac{\bar{\phi}}{|\phi|} \Theta_{\Gamma}\left(\left(\sigma_{n, N}\right)^{2}\right)
\end{aligned}
$$

where $\sigma_{n, N}(z)=1 / \sqrt{\pi} \sum_{k=1}^{N} \sqrt{k} x_{k}^{(n)} z^{k-1} d z$. Since $\left\{\Theta_{\Gamma}\left(\sigma_{n, N}^{2}\right)\right\}_{N=1}^{\infty}$ converges to $\phi$, we conclude that

$$
\left|\lim _{N \rightarrow \infty}\left(f_{n, N} \circ F_{\phi}\right)^{\prime}(0)\right|=\iint_{\Delta / \Gamma} \frac{\bar{\phi}}{|\phi|} \phi=\iint_{\Delta_{\mid} \Gamma}|\phi|=1 .
$$

Hence, from (6) and from the diagonal argument, we can take desired holomorphic functions of $T(\Gamma)$ satisfying (2). Considering the relation (1), we verify that the statement of Corollary 2.1 holds.

Next, we show :

LEMMA 3.3. Let $\Gamma$ be a cyclic Fuchsian group generated by a hyperbolic Möbius transformation. If $\phi \equiv A_{1}(\Delta, \Gamma)$ is a square of an Abelian differential for $\Gamma$, then the statements of Theorem 2.1. (a) and Corollary 2.1. hold on $\boldsymbol{D}(\phi)$. 
Proof of Lemma 3.3. Without loss of generality, we may consider the upper half plane $U$ instead of $\Delta$, and assume that $\Gamma$ is generated by $\gamma(z)=\lambda z(\lambda>1)$ and that $\|\phi\|_{U, \Gamma}=1$. We set

$$
\phi_{0}(z)=z^{-2} d z^{2} .
$$

Obviously, $\phi_{0} \cong A_{1}(U, \Gamma)$. It is easily seen that

$$
\Theta_{\Gamma}\left(\phi_{0}\right)=\phi_{0},
$$

where $\phi_{0}(z)=(1-\lambda)\{z(z-1)(z-\lambda)\}^{-1} d z^{2} \in A_{1}(U, 1)$. Since $\phi \in A_{1}(U, \Gamma)$ is regarded as a holomorphic quadratic differential on an annulus $A=U / \Gamma=\{w \in C: 1<|w|$ $<R\}, \phi / \phi_{0}$ is a square of a holomorphic function $g(w)=\sum_{k=-\infty}^{\infty} c_{k} w^{k}$ in $A$.

On the other hand, on $\Omega=\{1 \leqq|z|<\lambda\}$, a fundamental domain for $\Gamma$,

$$
\iint_{\Omega}|\phi|=\iint_{\Omega}\left|\frac{\phi}{\phi_{0}}\right|\left|\phi_{0}\right|<\infty
$$

and $\left|\phi_{0}\right|=\left|z^{-2} d z^{2}\right|$ is "bounded" in $\Omega$. Therefore,

$$
\iint_{A}|g(w)|^{2}|d w \wedge d \bar{w}|=2 \pi \sum_{k=-\infty}^{+\infty}\left|c_{k}\right|^{2} \frac{\left(R^{2 k+2}-1\right)}{k+1}<\infty .
$$

Hence, for $g_{l}(w)=\sum_{k=-l}^{l} c_{k} w^{k}(l \cong \boldsymbol{N})$

$$
\iint_{A}\left|g(w)-g_{l}(w)\right|^{2}|d w \wedge d \bar{w}| \longrightarrow 0 \quad(\text { as } l \rightarrow \infty) .
$$

Denote by $\tilde{g}_{l}(z)(z \in U)$ the lift of $g_{l}(w)$ on $U$, which is an automorphic function for $\Gamma$. Then, $\phi_{l}=\tilde{g}_{l}(z)^{2} \psi_{0}$ belongs to $A_{1}(U, 1)$ because $\tilde{g}_{l}$ is a bounded holomorphic function. Put

$$
\phi_{l, n}=\frac{1}{2 n+1} \sum_{\jmath=-n}^{n} \gamma_{*}^{\jmath} \psi_{l} \quad(n=1,2, \cdots),
$$

then $\Theta_{\Gamma}\left(\phi_{l, n}\right)=\Theta_{\Gamma}\left(\psi_{l}\right)$. According to a result by McMullen [10], [11],

$$
\lim _{n \rightarrow \infty}\left\|\phi_{l, n}\right\|_{U, 1}=\left\|\Theta_{\Gamma}\left(\phi_{l}\right)\right\|_{U / \Gamma, 1} .
$$

Since $\tilde{g}_{l}^{2}$ is an automorphic function for $\Gamma$, we have

$$
\begin{aligned}
\phi_{l, n}(z) & =\frac{1}{2 n+1} \sum_{\jmath=-n}^{n} \tilde{g}_{l}(z)^{2} \psi_{0}\left(\lambda^{\jmath} z\right) \lambda^{2 \jmath} \\
& =\frac{\tilde{g}_{l}(z)^{2}}{2 n+1} \sum_{\jmath=-n}^{n} \frac{1}{z}\left(\frac{\lambda^{\jmath}}{\lambda^{\jmath} z-1}-\frac{\lambda^{\jmath-1}}{\lambda^{\jmath-1} z-1}\right) d z^{2} \\
& =\frac{\tilde{g}_{l}(z)^{2}}{2 n+1} \frac{1}{z}\left(\frac{\lambda^{n}}{\lambda^{n} z-1}-\frac{\lambda^{-n-1}}{\lambda^{-n-1} z-1}\right) d z^{2} \\
& =\frac{\tilde{g}_{l}(z)^{2}}{2 n+1} \frac{1}{z} \frac{\lambda^{-n-1}-\lambda^{n}}{\left(\lambda^{n} z-1\right)\left(\lambda^{-n-1} z-1\right)} d z^{2} .
\end{aligned}
$$


Noting that $\tilde{g}_{l}^{2}(z)$ has zeros of even order only, we conclude that $\left\{\psi_{l, n}\right\}_{n=1}^{\infty}$ is an efficient sequence for $\Theta_{\Gamma}\left(\phi_{l}\right)$ with zeros of even order. Namely, $\phi_{l}:=$ $\Theta_{\Gamma}\left(\phi_{l}\right)=\tilde{g}_{l}^{2} \phi_{0}$ admits a sequence $\left\{\psi_{l, n}\right\}_{n=1}^{\infty}$ with zeros of even order only such that $\Theta_{\Gamma}\left(\phi_{l, n}\right)=\phi_{l}$ and that $\lim _{n \rightarrow \infty}\left\|\phi_{l, n}\right\|_{U, 1}=\left\|_{i} \phi_{l}\right\|_{U / \Gamma, 1}$.

We define a holomorphic mapping $f_{n, N}^{l}$ of $T(\Gamma)$ for $\phi_{l}$ and $\phi_{l, n}(n . N=$ $1,2, \cdots)$ as (5). Then by the same argument as in the proof of Lemma 3.2, we have

$$
\lim _{N \rightarrow \infty}\left(f_{n, N}^{l} \circ F_{\phi}\right)^{\prime}(0)=-\iint_{U / \Gamma} \frac{\phi}{|\phi|} \phi_{l}
$$

and

$$
\lim _{N \rightarrow \infty}\left\|f_{n, N}^{l}\right\|_{\infty} \leqq\left\|\phi_{l, n}\right\|_{U, 1} .
$$

By (7) we see that $\left\|\phi-\phi_{l}\right\|_{U, \Gamma \rightarrow 0}$ as $l \rightarrow \infty$. Thus, we have

$$
\left|\lim _{l, n \rightarrow \infty} \lim _{N \rightarrow \infty}\left(f_{n, N}^{l} \circ F_{\phi}\right)^{\prime}(0)\right|=\|\boldsymbol{\phi}\|_{U / \Gamma, 1}=1 \text {. }
$$

Therefore, the equality of the Carathéodory and the Teichmüller distances is proved. Similarly, we see that the statement of Corollary 2.1 holds on $\boldsymbol{D}(\phi)$. We have established the proof of Lemma 3.3.

We proceed to prove Theorem 2.1 (a). We have already shown the theorem in case $\Gamma$ is a hyperbolic cyclic group and $\phi \Subset A_{1}(\Delta, \Gamma)$ is a square of an Abelian differential for $\Gamma$. In case $\Gamma$ is a hyperbolic cyclic group but $\phi$ is not a square of any Abelian differential for $\Gamma, \phi$ is a square of an Abelian differential for $\Gamma^{2}=\left\langle\gamma^{2}\right\rangle$ because $\phi$ has zeros of even order only. Thus, the statements of Theorem 2.1 (a) and Corollary 2.1 hold for $\boldsymbol{D}(\phi) \subset T\left(\Gamma^{2}\right)$. By the same argument in the beginning of this section, we verify that the Carathéodory distance is the same as the Teichmüller distance on $\boldsymbol{D}(\phi) \subset T(\Gamma)$ as well as Corollary 2.1. Similarly, we also verify that if $\Gamma$ is a $\boldsymbol{Z}_{2}$ extension of hyperbolic cyclic group, then we have the desired results.

We assume that $\Gamma=\langle\gamma\rangle$ is a parabolic cyclic group. We may assume that $\Gamma$ is generated by a parabolic $\gamma$ with the fixed point $z=1$.

Let $p$ be an arbitrary point on $\boldsymbol{D}(\phi) \subset T(\Gamma)$ determined by Beltrami coefficients $t \bar{\phi} /|\phi|(t \in \Delta)$. We set

$$
\Delta_{c}=\left\{z \in \Delta ;\left|z-\frac{1+c}{2}\right|>\frac{1-c}{2}\right\} \quad \text { for } 0<c<1,
$$

the outside of horodisk tangent at $z=1$. Note that $\Delta_{c} \rightarrow \Delta$ as $c \rightarrow 1$. We denote by $G_{c}$ the Riemann mapping from $\Delta$ onto $\Delta_{c}$ with $G_{c}(0)=0$ and $G_{c}^{\prime}(0)>0$. Then, $\Gamma_{c}=\left(G_{c}\right)^{-1} \Gamma G_{c}$ is a hyperbolic cyclic group on $\Delta$ and $\phi_{(c)}=\phi^{\circ} G_{c}\left(G_{c}^{\prime}\right)^{2}$ is in $A_{1}\left(\Delta, \Gamma_{c}\right)$. Thus, $\phi_{(c)}$ is regarded as a holomorphic quadratic differential on $A_{c}=\Delta / \Gamma_{c}$ which is contained in a punctured disk $A_{1}=\Delta / \Gamma$. Since $G_{c}$ converges to the identity uniformly on every compact subset of $\Delta$ as $c \rightarrow 1$ (Carathéodory convergence theorem), $\phi_{(c)}(z) \rightarrow \phi(z)(c \rightarrow 1)$ as a holomorphic quadratic differential 
in $A_{c} \subset A_{1}$.

Since $\phi \in A_{1}(\Delta, \Gamma)$ has zeros with even order only, $\phi_{(c)}$ has also zeros of even order only. We can take a sequence of holomorphic functions $\left\{f_{N}^{(c)}\right\}_{N=1}^{\infty}$ from $\phi_{(c)}$ like (5) in the proof of Lemma 3.2. The functions $f_{N}^{(c)}(N=1,2, \cdots)$ are bounded holomorphic functions on $T(1)$ as well as on $T(\Gamma)$. Thus, $f_{N}^{(c)} \circ F_{\phi}$ is well defined for every $c$. Noting that $\left\|\phi_{(c)}\right\|_{\Delta / \Gamma_{c}, 1} \leqq\|\phi\|_{\Delta / \Gamma_{, 1}}=1$, we verify that $\lim _{c \rightarrow 1, N \rightarrow \infty}\left\|f_{N}^{(c)}\right\|_{\infty} \leqq 1$. Applying the same argument as in the proof of Lemma 3.2 and Lebesgue's dominated convergence theorem, we have

$$
\lim _{c \rightarrow 1, N \rightarrow \infty}\left|\left(f_{N}^{(c)} \circ F_{\phi}\right)^{\prime}(0)\right|=\left|\iint_{A_{1}} \frac{\phi}{|\phi|} \phi\right|=\|\phi\|_{\Delta / I^{\prime}, 1}=1
$$

The proof of (a) is now complete.

Proof of (b). First, we note:

Suppose that $\left\|\Theta_{\Gamma}\right\|=1$. It is shown (Ohtake [13] Theorem 1) that $\left\|\Theta_{\Gamma}\right\|=1$ if and only if $\Gamma$ satisfies one of the following conditions:

$\left(O_{1}\right)$ For any $r>0$, there exists a hyperbolic disk $D$ in $\Delta$ with radius $r$ such that

$$
D \cap \gamma D=\text { for all } \gamma \in \Gamma-\{1\} \text {. }
$$

$\left(\mathrm{O}_{2}\right)$ For any $\varepsilon>0$, there exists a hyperbolic element $\gamma \in \Gamma$ whose multiplier $\lambda$ satisfies $1<\lambda<1+\varepsilon$.

Suppose that $\Delta / \Gamma$ satisfies $\left(O_{1}\right)$. For a sequence $\left\{r_{n}\right\}_{n=1}^{\infty}$ of positive numbers with $\lim _{n \rightarrow \infty} r_{n}=\infty$, there exists a sequence $\left\{D_{n}\right\}_{n=1}^{\infty}$ of disks in $\Delta / \Gamma$ such that the radius of $D_{n}$ is greater than $r_{n}$ for each $n$. Then by the same argument as in [13] p. 59, we can take $\phi_{n} \in A_{1}(\Delta)(n=1,2, \cdots)$ such that $\phi_{n}$ has no zeros in $\Delta,\left\|\psi_{n}\right\|_{\Delta, 1}=1$,

$$
\lim _{n \rightarrow \infty}\left\|\Theta_{\Gamma}\left(\phi_{n}\right)\right\|_{\Delta / \Gamma, 1}=1
$$

and

$$
\lim _{n \rightarrow \infty} \iint_{D_{n}}\left|\Theta_{\Gamma}\left(\psi_{n}\right)\right|=1
$$

From the above formulae the sequence $\left\{\Theta_{\Gamma}\left(\phi_{n}\right)\right\}$ converges to 0 uniformly on compact subsets of $\Delta / \Gamma$. There exists a Beltrami differential $\mu$ for $\Gamma$ such that the sequence $\left\{\Theta_{\Gamma}\left(\psi_{n}\right)\right\}_{n=1}^{\infty}$ is a Hamilton sequence for $\mu$ (cf. Tanigawa [17]). Namely,

$$
\sup \left\{\left|\iint_{\Delta / \Gamma} \mu \varphi\right|:\|\varphi\|_{\Delta / \Gamma, 1} \leqq 1\right\}=\lim _{n \rightarrow \infty}\left|\iint_{\Delta / \Gamma} \mu \Theta_{\Gamma}\left(\phi_{n}\right)\right|=\|\mu\|_{\infty}
$$

Since

$$
\iint_{\Delta, \Gamma} \mu \Theta_{\Gamma}\left(\psi_{n}\right)=\iint_{\Delta} \pi^{*} \mu \psi_{n}
$$

we have 


$$
\left\|\pi^{*} \mu\right\|_{\infty} \geqq \sup \left\{\left|\iint_{\Delta} \pi^{*} \mu \psi\right|:\|\phi\|_{\Delta, 1} \leqq 1\right\} \geqq \lim _{n \rightarrow \infty}\left|\iint_{\Delta} \pi^{*} \mu \psi_{n}\right|=\left\|\pi^{*} \mu\right\|_{\infty},
$$

where $\pi: \Delta \rightarrow \Delta / \Gamma$ is a universal covering mapping. This implies that $\left\{\psi_{n}\right\}_{n=1}^{\infty}$ is still a Hamilton sequence for $\pi^{*} \mu$. It follows that $\mu$ and $\pi^{*} \mu$ are extremal Beltrami differentials on $\Delta / \Gamma$ and $\Delta$, respectively. Hence from Lemma 3.1, the Beltrami differential $\mu$ has the desired property.

Next, we suppose that $\Delta / \Gamma$ satisfies $\left(\mathrm{O}_{2}\right)$. Then, there exists a sequence of hyperbolic transformations $\left\{\gamma_{n}\right\}_{n=1}^{\infty}$ in $\Gamma$ such that their multiplies coverge to one. Using the same argument as in Ohtake [13] again, we can take $\phi_{n} \Leftarrow$ $A_{1}(\Delta, 1)$ with no zeros in $\Delta(n=1,2, \cdots)$ such that $\Phi_{n}=\Theta_{\Gamma}\left(\phi_{n}\right) \boxminus A_{1}(\Delta, \Gamma)$ satisfis $\lim _{n \rightarrow \infty}\left\|\Phi_{n}\right\|_{\Delta / \Gamma, 1}=1$ and the weight of $\Phi_{n}$ almost concentrates in a (maximal) collar for $\Gamma$. By the same argument as above, we can construct a Beltrami differential $\mu$ for $\Gamma$ with the desired property.

Conversely, Suppose that there exists a point $\varphi \leqq T(\Gamma)$ such that

$$
\rho(0, \kappa(\varphi))=\tau_{T(1)}(0, \varphi)=\tau_{T(\Gamma)}(0, \varphi) .
$$

Let $\mu$ be an extremal Beltrami differential for $\mu \in T(\Gamma)$. It follows from Lemma 3.1 that

$$
\sup \left|\iint_{\Delta} \mu \phi\right|=\sup \left|\iint_{\Delta_{1} \Gamma} \mu \Theta_{\Gamma}(\phi)\right|=\|\mu\|_{\infty} .
$$

Since $\left\|\Theta_{\Gamma}\right\| \leqq 1$, it means that $\left\|\Theta_{\Gamma}\right\|=1$.

\section{Proof of Theorem 2.2}

For $\gamma \subseteq \Gamma$ we set $\gamma_{\varphi}=W_{\varphi} \gamma\left(W_{\varphi}\right)^{-1}$. From the definition of Grunsky coefficients

$$
\log \frac{W_{\varphi}(\gamma(z))-W_{\varphi}(\gamma(\zeta))}{\gamma(z)-\gamma(\zeta)}=-\sum_{\imath, j \geqq 1} b_{i j}(\varphi) \gamma(z)^{-\imath} \gamma(\zeta)^{-\jmath} \text {. }
$$

The left hand side is

$$
\begin{aligned}
\log & \frac{W_{\varphi}(\gamma(z))-W_{\varphi}(\gamma(\zeta))}{\gamma(z)-\gamma(\zeta)}=\log \frac{\gamma_{\varphi}\left(W_{\varphi}(z)\right)-\gamma_{\varphi}\left(W_{\varphi}(\zeta)\right)}{\gamma(z)-\gamma(\zeta)} \\
& =\log \frac{\gamma_{\varphi}\left(W_{\varphi}(z)\right)-\gamma_{\varphi}\left(W_{\varphi}(\zeta)\right)}{W_{\varphi}(z)-W_{\varphi}(\zeta)} \cdot \frac{W_{\varphi}(z)-W_{\varphi}(\zeta)}{z-\zeta} \cdot \frac{z-\zeta}{\gamma(z)-\gamma(\zeta)} \\
& =\log \frac{\gamma_{\varphi}\left(W_{\varphi}(z)\right)-\gamma_{\varphi}\left(W_{\varphi}(\zeta)\right)}{W_{\varphi}(z)-W_{\varphi}(\zeta)}+\log \frac{W_{\varphi}(z)-W_{\varphi}(\zeta)}{z-\zeta}+\log \frac{z-\zeta}{\gamma(z)-\gamma(\zeta)}
\end{aligned}
$$

Using the formula

$$
\gamma(z)-\gamma(\zeta)=\gamma^{\prime}(z)^{1 / 2} \gamma^{\prime}(\zeta)^{1 / 2}(z-\zeta)
$$

we verify that 


$$
\frac{1}{2} \log \frac{\gamma_{\varphi}^{\prime}\left(W_{\varphi}(z)\right) \gamma_{\varphi}^{\prime}\left(W_{\varphi}(\zeta)\right)}{\gamma^{\prime}(z) \gamma^{\prime}(\zeta)} \sum_{\imath, j \geq 1} b_{\imath \jmath}(\varphi) z^{-\imath \zeta^{-\jmath}}=-\sum_{\imath, j \geq 1} b_{\imath \jmath}(\varphi) \gamma(z)^{-\imath} \gamma(\zeta)^{-\jmath}
$$

Taking the limit as $z, \zeta \rightarrow \infty$, we have

$$
2 \log \frac{c}{c_{\varphi}}=-\sum_{\imath, j \geqq 1} b_{\imath \jmath}(\varphi)\left(\frac{a}{c}\right)^{-\imath}\left(\frac{a}{c}\right)^{-\jmath},
$$

where $\gamma(z)=(a z+b) /(c z+d) \quad(a d-b c=1)$ and $\gamma_{\varphi}(z)=\left(a_{\varphi} z+b_{\varphi}\right) /\left(c_{\varphi} z+d_{\varphi}\right)\left(a_{\varphi} d_{\varphi}-\right.$ $\left.b_{\varphi} c_{\varphi}=1\right)$. Put $x_{n}=n^{-1 / 2}(c / a)^{n}(n=1,2, \cdots)$ in the definition of the Grunsky norm, then we have

$$
\left|2 \log \frac{c}{c_{\varphi}}\right| \leqq \kappa(\varphi) \sum_{k=1}^{\infty} \frac{1}{k}\left|\frac{c}{a}\right|^{2 k}=-\kappa(\varphi) \log \left(1-\left|\frac{c}{a}\right|^{2}\right) .
$$

Here, we note that $|c / a|=|\gamma(\infty)|^{-1}<1$ because $\gamma(\Delta)=\Delta$. Hence we have

$$
\left(1-\left|\frac{c}{a}\right|^{2}\right)^{\kappa(\varphi) / 2} \leqq\left|\frac{c}{c_{\varphi}}\right| \leqq\left(1-\left|\frac{c}{a}\right|^{2}\right)^{-\kappa(\varphi) / 2}
$$

and

$$
|c|^{-1}\left(1-\left|\frac{c}{a}\right|^{2}\right)^{\kappa(\varphi) / 2} \leqq\left|c_{\varphi}\right|^{-1} \leqq|c|^{-1}\left(1-\left|\frac{c}{a}\right|^{2}\right)^{-\kappa(\varphi) / 2} .
$$

Since $\gamma$ preserves the unit circle, it is easy to see that $|a|^{-2}=\left(1-|c / a|^{2}\right)$. On the other hand, since $\Gamma$ acts discontinuously on $\Sigma$ and $|a / c|=|\gamma(\infty)|$, for every number $r>1,1<|a / c|<r$ for all but finite number of elements of $\Gamma$. Thus except for finite number of elements,

$$
r^{-\kappa(\varphi)}|c|^{-1-\kappa(\varphi)} \leqq\left|c_{\varphi}\right|^{-1} \leqq r^{\kappa(\varphi)}|c|^{-1+\kappa(\varphi)}
$$

Summing up them, we obtain the desired inequality for critical exponents.

\section{Examples}

First, we give an example of a Teichmüller disk in $T(1)$ defined by a quadratic differential with a simple zero on which $c_{T(1)}(\cdot, \cdot)=\tau_{T(1)}(\cdot, \cdot)$. In particular, for each point $\varphi$ in the Teichmüller disk, $\rho(0, \kappa(\varphi))<c_{T(1)}(0, \varphi)=$ $\tau_{T(1)}(0, \varphi)$ by the result of Krushkal'.

Example 5.1. Let $D=D(2 \pi z / 3)$ be the Teichmüller disk in $T(1)$ determined by the quadratic differential $\phi(z)=2 \pi z / 3 \in A_{1}(\Delta, 1)$, namely, setting $\mu_{0}=\bar{z} /|z|$, the Teichmüller disk $D$ is defined by $D=\left\{\left[w \mu_{0}\right] ; w \Subset \Delta\right\}$, where $\left[w \mu_{0}\right]$ denotes the point of $T(1)$ determined by the Beltrami coefficient $w \mu_{0}$. Then for any points $\varphi_{1}$ and $\varphi_{2}$ in $D, c_{T(1)}\left(\varphi_{1}, \varphi_{2}\right)=\tau_{T(1)}\left(\varphi_{1}, \varphi_{2}\right)$.

We shall show this in the following. For each Beltrami coefficient $\mu$ in $\Delta$, we extend $\mu$ to the whole plane by setting 0 outside $\Delta$. Let $w^{\mu}$ be the quasi- 
conformal automorphism of the Riemann sphere with Beltrami coefficient $\mu$ which is normalized at $\infty$ so that

near $\zeta=\infty$. Let

$$
w^{\mu}(\zeta)=\zeta+O\left(|\zeta|^{-1}\right)
$$

$$
w^{\mu}(\zeta)=\zeta+\sum_{n=1}^{\infty} b_{n}(\mu) \zeta^{-n}
$$

be the expansion at $\zeta=\infty$. Then, as is well-known, $b_{n}(\mu)$ is a holomorphic function on $T(1)$ for each $n$. By Schiffer's inequality (cf. Duren [4] Theorem 4.6), $\left|b_{2}(\mu)\right|<2 / 3$ for any Beltrami coefficient $\mu$, hence the assignment $\mu \mapsto$ $3 b_{2}(\mu) / 2$ induces a bounded holomorphic function $F([\mu])=3 b_{2}(\mu) / 2$ on $T(1)$ with $\|F\|_{\infty}=1$, where $[\mu]$ stands for the point in $T(1)$ determined by $\mu$.

Now, let $f: \Delta \rightarrow D$ be the holomorphic mapping defined by $f(w)=\left[w \mu_{0}\right]$. We shall verify that the mapping $F \circ f: \Delta \rightarrow \Delta$ is an isomorphism of $\Delta$. We use the variational formula

$$
w^{\mu}(\zeta)=\zeta-\frac{1}{\pi} \iint_{\Delta} \frac{\mu(z) d x d y}{z-\zeta}+O\left(\|\mu\|_{\infty}^{2}\right),
$$

where $z=x+i y, \zeta \equiv \Sigma$ and $\mu$ is a Beltrami coefficient on $\Delta$. We rewrite this in the form;

$$
\begin{aligned}
w^{\mu}(\zeta) & =\zeta+\frac{1}{\pi} \iint_{\Delta} \mu(z) \sum_{n=1}^{\infty} \zeta^{-n} z^{n-1} d x d y+O\left(\|\mu\|_{\infty}^{2}\right) \\
& =\zeta+\sum_{n=1}^{\infty} \zeta^{-n} \frac{1}{\pi} \iint_{\Delta} \mu(z) z^{n-1} d x d y+O\left(\|\mu\|_{\infty}^{2}\right) .
\end{aligned}
$$

Comparing this expansion with (11),

$$
b_{n}(\mu)=\frac{1}{\pi} \iint_{\Delta} \mu(z) z^{n-1} d x d y+O\left(\|\mu\|_{\infty}^{2}\right) .
$$

Hence the derivative of the holomorphic function $F \circ f$ at the origin is

$$
\left|(F \circ f)^{\prime}(0)\right|=\frac{3}{2 \pi}\left|\iint_{\Delta} \frac{\bar{z}}{|z|} z d x d y\right|=1 .
$$

Therefore, we have shown $c_{T(1)}(\cdot, \cdot)=\tau_{T(1)}(\cdot, \cdot)$ on $D$.

Next, we construct an example in relation to Theorem 2.1 (b). By the argument of the proof of Lemma 3.1, for a point $\varphi \in T(\Gamma)$ if the equality $\rho(0, \kappa(\varphi))=\tau_{\left.T_{(\Gamma)}\right)}(0, \varphi)$ is valid then $\varphi$ corresponds to an extremal Beltrami coefficient $\mu$ on $\Delta$ with a Hamilton sequence $\left\{\psi_{n}\right\} \subset A_{1}(\Delta, \Gamma)$ in the form $\phi_{n}=$ $\Theta_{\Gamma}\left(\tilde{\psi}_{n}\right)$ with $\tilde{\psi}_{n} \in A_{1}(\Delta, 1)$ such that all of the zeros of $\tilde{\psi}_{n}$ are of even order for each $n$ and $\tau_{T(\Gamma)}(0, \varphi)=\tau_{T(1)}(0, \varphi)$.

Now, conversely, assume that $\varphi \equiv T(\Gamma)$ satisfies $\tau_{T(\Gamma)}(0, \varphi)=\tau_{T(1)}(0, \varphi)$ and that $\varphi$ is represented by an extremal Beltrami differential $\mu$ for $\Gamma$ with a 
Hamilton sequence of the form $\left\{\Theta_{\Gamma}\left(\tilde{\psi}_{n}\right)\right\}$, where $\tilde{\psi}_{n} \in A_{1}(\Delta, 1)$ has no zeros of odd order (here, $\left\|\tilde{\psi}_{n}\right\|$ is not necessarily assumed to tend to 1 , while $\left\|\Theta_{\Gamma}\left(\tilde{\psi}_{n}\right)\right\|_{\Delta / \Gamma, 1}$ $\leqq 1$ from the definition of Hamilton sequence.) Does it follow that $\rho(0, \kappa(\varphi))$ $=\tau_{T(\Gamma)}(0, \varphi)$ ? The answer is no, since $\mu$ may have several essentially different Hamilton sequences, some of them may have inverse images via $\Theta_{\Gamma}$ with norms tending to 1 , others may have inverse images via $\Theta_{\Gamma}$ without zeros of odd order, and these conditions need not occur at the same time. We construct a counterexample as follows.

Example 5.2. There exists a Fuchsian group $\Gamma \neq 1$ and a point $\varphi \leqq T(\Gamma)$ such that

$$
\rho(0, \kappa(\varphi))<\tau_{T\left(\Gamma^{\prime}\right)}(0, \varphi)=\tau_{T(1)}(0, \varphi),
$$

while $\varphi$ is represented by an extremal Beltrami differential $\mu$ with a Hamilton sequence of the form $\left\{\Theta_{\Gamma}\left(\tilde{\psi}_{n}\right)\right\}$, where $\tilde{\psi}_{n}$ has no zeros of odd order.

Let $S_{0}$ be a compact Riemann surface and $S^{\prime}$ be a covering surface of $S_{0}$ whose covering group is a cyclic group of infinite order generated by a conformal automorphism $f: S^{\prime} \rightarrow S^{\prime}$. Divide $S^{\prime}$ by a smooth dividing curve $C$ into two ends $e_{1}^{\prime}$ and $e_{2}^{\prime}$ such that $f e_{2}^{\prime} \subset e_{2}^{\prime}$. There exists a sequence of smooth nondividing curves $\left\{C_{m}\right\}$ in $e_{1}^{\prime}$ so that $e_{1}^{\prime}-\bigcup_{m=1}^{\infty} C_{m}$ is conformally equivalent to an annulus. We set $S=S^{\prime}-\cup_{m=1}^{\infty} C_{m}, e_{1}=e_{1}^{\prime}-\bigcup_{m=1}^{\infty} C_{m}$ and $e_{2}=e_{2}^{\prime}$. The Riemann surface $S$ has two ends $e_{1}$ and $e_{2}$, and it is easy to see that $e_{1}$ satisfies condition $\left(O_{1}\right)$ in the proof of Lemma 3.3, while $e_{2}$ satisfies neither $\left(O_{1}\right)$ nor $\left(O_{2}\right)$. We shall construct a Beltrami differential $\mu_{0}$ on $S$ which has the desired property.

Let $\Gamma$ and $\Gamma^{\prime}$ be Fuchsian groups acting on $\Delta$ with $\Delta / \Gamma=S$ and $\Delta / \Gamma^{\prime}=S^{\prime}$. We identify $A_{1}(\Delta, \Gamma)$ and the space $A_{1}(S)$ of integrable holomorphic quadratic differentials on $S$, and in the same way $A_{1}\left(\Delta, \Gamma^{\prime}\right)$ and $A_{1}\left(S^{\prime}\right)$. We construct two sequences in the closed unit ball of $A_{1}(S)$ whose weights converge to ideal boundaries determined by $e_{1}$ and $e_{2}$ respectively.

First, fix a quadratic differential $\tilde{\phi}^{(2)} \in A_{1}(\Delta, 1)$ without zeros of odd order and put $\psi^{(2)}=\Theta_{\Gamma^{\prime}}\left(\tilde{\psi}^{(2)}\right) /\left\|\Theta_{\Gamma^{\prime}}\left(\tilde{\psi}^{(2)}\right)\right\|$ and $\phi_{n}^{(2)}=\left(f^{-n}\right)^{*}\left(\psi^{(2)}\right)$. Then there exists a subsequence $\left\{\phi_{n}^{(2)}\right\}$ of $\left\{\phi_{n}^{(2)}\right\}$ and a sequence of disjoint compact subsets $\left\{K_{j}\right\}$ in $e_{2}$ such that $\lim _{\jmath \rightarrow \infty} \iint_{K_{j}}\left|\phi_{n_{j}}^{(2)}\right|=1$ and $\bigcap_{k=1}^{\infty} \cup_{\jmath=k}^{\infty} K_{\jmath}=\varnothing$. In fact, let $\phi_{n_{1}}^{(2)}=\phi^{(2)}$ and $K_{1}$ be an arbitrarily fixed compact set of $e_{2}$. There exists a compact set $F_{2}$ in $S^{\prime}$ such that $\iint_{F_{2}}\left|\psi^{(2)}\right|>1-1 / 2$. Take a positive integer $n_{2}$ so that $f^{n_{2}}\left(F_{2}\right)$ is contained in $e_{2}$ and disjoint from $K_{1}$. Put $K_{2}=f^{n_{2}}\left(F_{2}\right)$, and we have $\iint_{K_{2}}\left|\phi_{n_{2}}^{(2)}\right|=\iint_{F_{2}}\left|\psi^{(2)}\right|>1-1 / 2$. Inductively, we can take a compact set $F_{J}$ in $S^{\prime}$ and a positive integer $n_{\jmath}$ such that $K_{\jmath}=f^{n_{j}}\left(F_{\jmath}\right)$ is disjoint from $K_{1} \cup \cdots \cup K_{\jmath-1}$ 
and $\iint_{K_{j}}\left|\psi_{n_{\jmath}}^{(2)}\right|=\iint_{F_{j}}\left|\psi^{(2)}\right|>1-1 / 2^{\jmath-1}$. Restrict each $\phi_{n_{\jmath}}^{(2)} \in A_{1}\left(S^{\prime}\right)$ to $S$ and denote the restriction by the same symbol.

Next, since the ideal boundary of $e_{1}$ is a free border, we can take a sequence of disjoint hyperbolic disks $D$, with hyperbolic radius $\mathcal{J}$ whose centers accumulates only to the ideal boundary. Let $E$ be the Dirichlet fundamental region in $\Delta$ for $\Gamma$ centered at 0 . Lift each $D$, and $K$, on $E$ and denote the lifts by $\tilde{D}$, and $\tilde{K}$, respectively and let $p$, denote the center (in hyperbolic geometry) of $\tilde{D}_{\jmath}$. Put $\tilde{\psi}^{(1)}(z)=2 \pi z / 3$ for $z \in \Delta$ and $\tilde{\psi}_{j}^{(1)}=\gamma_{j}^{*} \tilde{\psi}^{(1)}$, where $\gamma_{j}(z)=$ $\left(z-p_{j}\right) /\left(1-\bar{p}_{j} z\right)$. Then by the argument of Ohtake [13], $\lim _{\jmath \rightarrow \infty}\left\|\Theta_{\Gamma} \tilde{\psi}_{j}^{(1)}\right\|_{S, 1}=1$ and $\lim _{\jmath \rightarrow \infty} \iint_{D_{j}}\left|\Theta_{\Gamma} \tilde{\psi}_{j}^{(1)}\right|=1$.

Now, set $\Theta_{\Gamma} \tilde{\psi}_{j}^{(1)}=\phi_{j}^{(1)}$, and define a Beltrami differential $\mu_{0}$ on $S$ by

$$
\mu_{0}= \begin{cases}\bar{\psi}_{j}^{(1)} /\left|\phi_{j}^{(1)}\right|, & \text { in } D_{\jmath} \\ \bar{\psi}_{n_{j}}^{(2)} /\left|\psi_{n_{j}}^{(2)}\right|, & \text { in } K_{\jmath} \\ 0, & \text { elsewhere }\end{cases}
$$

Then for each $\lambda \Subset \Delta$ the Beltrami coefficient $\lambda \mu_{0}$ is an extremal Beltrami differential on $S$ with Hamilton sequences $\left\{\phi_{j}^{(1)}\right\}$ and $\left\{\psi_{n_{j}}^{(2)}\right\}$. The Beltrami differential $\mu_{0}$ is lifted on $\Delta$, which will be denoted by the same symbol.

Let $\varphi_{\lambda} \equiv T(\Gamma)$ denote the point determined by $\lambda \mu_{0}$ for $\lambda \equiv \Delta$. We shall show that these points $\lambda \mu_{0}$ have the desired property. First, note that $\tau_{T(\Gamma)}\left(0, \phi_{\lambda}\right)=$ $\tau_{T(1)}\left(0, \phi_{\lambda}\right)$, since $\lambda \mu_{0}$ has Hamilton sequence $\left\{\tilde{\phi}_{j}^{(1)}\right\}$ and $\left\{\phi_{j}^{(1)}\right\}$. The Beltrami coefficient $\lambda \mu_{0}$ also has Hamilton sequence $\left\{\psi_{n j}^{(2)}\right\}$, which is the image by $\Theta_{\Gamma}$ of a sequence in $A_{1}(\Delta, 1)$, with no zeros of odd order. Now we show $\rho(0, \kappa(\varphi))<$ $\tau_{\left.T_{(\Gamma)}\right)}(0, \varphi)$ by contradiction. If $\rho\left(0, \kappa\left(\varphi_{\lambda}\right)\right)=\tau_{\left.T_{(}\right)}\left(0, \varphi_{\lambda}\right)$, then by the argument of the proof of Lemma 3.1, the Beltrami coefficient $\lambda \mu_{0}$ is extremal not only in the equivalence class in $T(\Gamma)$ but also in the equivalence class in $T(1)$, and has a Hamilton sequence $\left\{\phi_{n}\right\} \subset A_{1}(\Delta, 1)$ with no zeros of odd order such that

$$
\lim _{n \rightarrow \infty}\left|\iint_{\Delta} \mu_{0} \phi_{n}\right|=\lim _{n \rightarrow \infty}\left|\sum_{\gamma \in \Gamma} \iint_{S} \mu_{0} \gamma^{*} \phi_{n}\right|=\lim _{n \rightarrow \infty} \sum_{\gamma \in \Gamma} \iint_{S}\left|\gamma^{*} \phi_{n}\right|=1 \text {. }
$$

On the other hand,

$$
\begin{aligned}
\left|\iint_{\Delta} \mu_{0} \phi_{n}\right| & \leqq \sum_{j=1}^{\infty} \sum_{\gamma \in \Gamma}\left|\iint_{\gamma \tilde{D}_{j}} \mu_{0} \phi_{n}\right|+\left|\sum_{j=1}^{\infty} \sum_{\gamma \in \Gamma} \iint_{\gamma \tilde{K}_{j}} \mu_{0} \phi_{n}\right| \\
& =\sum_{j=1}^{\infty} \sum_{\gamma \in \Gamma}\left|\iint_{\tilde{D}_{j}} \gamma^{*} \mu_{0} \gamma^{*} \phi_{n}\right|+\left|\iint_{e_{2}} \mu_{0} \Theta_{\Gamma}\left(\phi_{n}\right)\right| \\
& =\sum_{j=1}^{\infty} \sum_{\gamma \in \Gamma}\left|\iint_{\tilde{D}_{j}} \mu_{0} \gamma^{*} \phi_{n}\right|+\left|\iint_{e_{2}} \mu_{0} \Theta_{\Gamma}\left(\phi_{n}\right)\right|
\end{aligned}
$$




$$
\begin{aligned}
& =\sum_{j=1}^{\infty} \sum_{\gamma \in \Gamma}\left|\iint_{\tilde{D}_{\jmath}} \gamma_{J}^{*} \frac{\bar{z}}{|z|} \gamma^{*} \phi_{n}\right|+\left|\iint_{e_{2}} \mu_{0} \Theta_{\Gamma}\left(\phi_{n}\right)\right| \\
& =\sum_{j=1}^{\infty} \sum_{\gamma \in \Gamma}\left|\iint_{(\rho(0, z)<\jmath)} \frac{\bar{z}}{|z|}\left(\gamma_{J}^{-1}\right)^{*} \gamma^{*} \phi_{n}\right|+\left|\iint_{e_{2}} \mu_{0} \Theta_{\Gamma}\left(\phi_{n}\right)\right| .
\end{aligned}
$$

Here, it is easy to see (cf. Krushkal' [7]) that

$$
\begin{aligned}
\sup _{\phi} \frac{\left|\iint_{\{\rho(0, z)<j\}}(\bar{z} /|z|) \phi\right|}{\iint_{\{\rho(0, z)<j\}}|\phi|} & =\sup _{\|\phi\|_{\{\rho(0, z)<\jmath\}=1}}\left|\iint_{(\rho(0, z)<\jmath\}} \frac{\bar{z}}{|z|} \phi\right| \\
& \leqq \sup _{\|\phi\|=1}\left|\iint_{\Delta} \frac{\bar{z}}{|z|} \phi\right| \\
& \leqq 2 \sqrt{2} / 3<1,
\end{aligned}
$$

where the supremum is taken over the subclass of $A_{1}(\Delta, 1)$ consisting of all elements with no zeros of odd order. (The first inequality is shown by rescaling.) Hence we have

$$
\begin{aligned}
\left|\iint_{\Delta} \mu_{0} \phi_{n}\right| & \leqq \frac{2 \sqrt{2}}{3} \sum_{j=1}^{\infty} \sum_{\gamma \in \Gamma} \iint_{\tilde{D}_{j}}\left|\gamma^{*} \phi_{n}\right|+\left|\iint_{e_{2}} \mu_{0} \Theta_{\Gamma}\left(\phi_{n}\right)\right| \\
& =\frac{2 \sqrt{2}}{3} \sum_{j=1}^{\infty} \sum_{\gamma \in \Gamma} \iint_{\gamma \tilde{D}_{j}}\left|\phi_{n}\right|+\left|\iint_{e_{2}} \mu_{0} \Theta_{\Gamma}\left(\phi_{n}\right)\right|
\end{aligned}
$$

In view of (14) and the assumption that the $\operatorname{discs}\left\{\gamma \widetilde{D}_{j}\right\}$ are pairwise disjoint and $\lim _{n \rightarrow \infty}\left\|\phi_{n}\right\|_{\Delta, 1}=1$, it follows that

$$
\lim _{n \rightarrow \infty}\left|\iint_{e_{2}} \mu_{0} \Theta_{\Gamma}\left(\phi_{n}\right)\right|=\lim _{n \rightarrow \infty} \iint_{e_{2}} \mid \Theta_{\Gamma}\left(\phi_{n}\right)=1 .
$$

However, the end $e_{2}$ satisfies neither condition $\left(O_{1}\right)$ nor $\left(O_{2}\right)$, hence by the argument of Ohtake [13]

$$
\sup _{n}\left\|\Theta_{\Gamma}\left(\phi_{n}\right)\right\|_{\Delta / \Gamma, 1} /\left\|\phi_{n}\right\|_{\Lambda, 1}<1 .
$$

From (15), it follows that $\lim \inf \left\|\phi_{n}\right\|_{\Delta, 1}>1$. This contradicts the assumption $\lim _{n \rightarrow \infty}\left\|\phi_{n}\right\|_{\Delta_{1}}=1$.

Added in Proof. We can show for elementary groups $\Gamma_{p}=\left\langle\gamma_{p}\right\rangle$, where $\gamma_{p}(z)=e^{2 \pi \imath / p} z$ for $p \geqq 2$, that $c_{T\left(\Gamma_{p}\right)}=\tau_{T\left(\Gamma_{p}\right)}$ on $\boldsymbol{D}\left(\bar{z}^{p-2} /|z|^{p-2}\right)$ in the same way as Example 5.1. 


\title{
REFERENCES
}

[1] L.V. AhLFors, The complex analytic structure of the space of closed Riemann surfaces in "Analytic Functions", 45-66, 1960.

[2] L. Bers, On boundaries of Teichmüller spaces and on kleinian groups l, Ann. of Math. 91 (1970), 570-600.

[3] L. Bers, Finite dimensional Teıchmüller spaces and generalizations, Bull. Amer. Math. Soc. (N. S.) 5 (1981), 131-172.

[4] P.L. Duren, Univalent Functions, A Series of Comprehensive Studies in Mathematics 259, Springer-Verlag New York Berlin Heidelberg Tokyo, 1983.

[5] Y. IMAYOSH AND H. Shiga, A finiteness theorem for holomorphic families of Riemann surfaces in "Holomorphic Functions and Moduli II", Springer-Verlag New York Berlin Heidelberg London Paris Tokyo, 207-219, 1988.

[6] I. KRA, The Carathéodory metric on abelian Teichmüller disks, J. Analyse Math. 40 (1981), 129-143.

[7] S.L. KrushKal', On the Grunsky coefficient conditions, Siberian Math. J. 28 (1987), 138-145.

[8] S. L. KRUSHKAL', Grunsky coefficient inequalities, Carathéodory metric and extremal quasiconformal mappings, Comment. Math. Helvetici 64 (1989), 650-660.

[9] O. Lehto, Univalent Functions and Teichmüller Spaces, Graduate Texts in Mathematics 109, Springer-Verlag New York Berlin Heidelberg London Paris Tokyo, 1986.

[10] C. McMullen, Amenability, Poincaré series and quasiconformal maps, Invent. Math. 97 (1989), 95-127.

[11] C. McMullen, Ameinable coverings of complex manifolds and holomorphic probability measures, Invent. Math. 110 (1992), 29-37.

[12] H. OHTAKE, Lifts of extremal quasiconformal mappings of arbitrary Riemann surfaces, J. Math. Kyoto Univ. 22 (1982), 191-200.

[13] H. Oнtake, On the norm of Poincaré series operator for a universal covering group, J. Math. Kyoto Univ. 32 (1992), 57-72.

[14] H. Shiga, On analytic and geometric properties of Teichmüller spaces, J. Math. Kyoto Univ. 24 (1984), 441-452.

[15] H. SHIGA, Characterization of quasi-disks and Teichmüller spaces, Tôhoku Math. J. 37 (1985), 541-552.

[16] D. Sullivan, Entropy, Hausdorff measures old and new, and limit sets of geometrically finite Kleinian grovps, Acta Math. 153 (1984), 259-277.

[17] H. Tanigawa, Holomorphic families of geodesic discs in infinite dimensional Teichmüller spaces, Nagoya Math. J. 127 (1992), 117-128.

[18] I. V. Zuravlev, Univalent functions and Teichmüller spaces, Soviet Math. Dokl. 21 (1980), 252-255.

\author{
Department of Mathematics \\ Tokyo Institute of TECHNOLOgy \\ Department of Mathematics \\ NAGOYA UNIVERSITY
}

